\title{
A Numerical Investigation of Clustered Spheres Separating in Mach 20 Flow
}

\author{
Thomas J. Whalen ${ }^{\mathrm{a}}$ and Stuart J. Laurence ${ }^{\mathrm{b}}$ \\ University of Maryland, College Park, MD, 20742 \\ Ralf Deiterding ${ }^{\mathrm{c}}$ \\ University of Southampton, Highfield, Southampton, SO17 1BJ, UK
}

In the current study, the separation of equal-sized spheres from initial touching configurations at Mach 20 is examined through numerical simulations using a coupled CFD/FEA solver. Comparison of computational results with free-flight wind tunnel trajectories is conducted, and modest dependence on viscosity is observed, namely in the form of enhanced drag, although errors in lateral velocity are deemed low enough for computational results to be considered representative of actual sphere motions. A survey of two initially touching spheres at various alignment angles reveals a bimodal distribution of separation behaviors, with a stable region of persistent contact detected. From a different survey of four spheres arranged in the form of a tetrahedron, the final lateral velocity is correlated to initial polar positioning in the cluster, and a common assumption of purely radial spreading is supported by minimal changes in azimuthal positioning. Finally, a reduced parameterization of tetrahedron orientation allows for characterization of collective separation velocities and center-of-mass motion.

Nomenclature

$\begin{array}{ll}A & \text { Asymmetry index } \\ B & \text { Bluntness index } \\ C & \text { Aerodynamic separation coefficient } \\ C_{D} & \text { Drag coefficient } \\ C_{L} & \text { Lateral force coefficient } \\ p_{\infty} & \text { Freestream pressure } \\ r & \text { Sphere radius } \\ t & \text { Time } \\ T_{\infty} & \text { Freestream temperature } \\ u_{\infty} & \text { Freestream velocity } \\ V_{T} & \text { Lateral sphere velocity } \\ V_{T} & \text { Collective lateral velocity of cluster } \\ \vec{x} & \text { Position vector } \\ x & \text { Streamwise coordinate } \\ \theta & \text { Polar alignment angle } \\ \rho_{\infty} & \text { Freestream density } \\ \phi & \text { Azimuthal angle } \\ l & \text { Nondimensional quantity }\end{array}$

${ }^{a}$ Graduate Student, NASA Space Technology Research Fellow, Department of Aerospace Engineering, AIAA Student Member.

b Associate Professor, Department of Aerospace Engineering, AIAA Senior Member.

c Associate Professor, School of Engineering, Aerodynamics and Flight Mechanics Research Group. 


\section{Introduction}

Aerodynamic entry of meteors commonly results in fracture of a progenitor body into smaller fragments, and the precise fragmentation process holds significant implications for potential ground damage [1]. While the onset of successive fragmentation occurs when the dynamic pressure of the hypersonic flow exceeds material strength of the meteor, aerodynamic interactions between nearby bodies primarily determine the separation behavior and eventual trajectories that arise. Characterization of the separation aerodynamics is thus critical to risk assessment. Initial efforts to model the separation of two bodies were undertaken by Passey and Melosh [2], who proposed a bow-shock interference model in which the transverse separation velocity of a secondary body is governed by an effective separation constant, $C$, estimated to a value between 0.03 and 2.25 . Later studies sought to provide more physically consistent support for the use of the separation constant; simulations of separating identical bodies conducted in Artem'eva and Shuvalov [3, 4] found values of 0.2 and 1.0 for two hemicylinders and numerous cubes, respectively, while also describing the attractive nature of flow behind the primary bow shock. Laurence et al. [5] examined the separation of spherical fragments of unequal size at various initial alignments and noted that the scaling law of Passey and Melosh is ill-suited to modeling more complex separation scenarios; in particular, they find highly augmented repulsion due to shock-wave surfing, a phenomenon in which aerodynamic forces cause the trajectory of secondary body to follow the bow shock of the primary. The current state-of-the-art for separation modeling is given by the fragment-cloud model of Wheeler et al. [6], in which a fragmenting body splits into a number of discrete fragments and a debris cloud deforming under the dispersion law proposed by Hills and Goda [7]. When integrated into the Probabalistic Asteroid Impact Rick of Mathias et al. [1], the model reproduces the energy deposition curve from the Chelyabinsk impact well, but the lack of underlying physical basis is problematic. More recently, Register et al. [8] have created a viscous-corrected simulation-derived database to seed a separation model of the two-body interaction. They divide the trajectories into distinct zones based on spherical radius ratio and initial positions, with nondimensionalization of important parameters providing a basis for predicting cloud dispersion. Despite clear improvements over previous methods, this more physically grounded modeling considers fragmentation into bodies that interact aerodynamically only with the primary body and not with each other, while also neglecting trajectory-altering collisions between bodies.

Given the significant uncertainties in the structural composition of meteors, exactly modeling their fragmentation into large numbers of discrete fragments is a complex problem. In this work, we attempt to describe general trends and provide a basis for future modeling efforts derived from experiments and computations of clustered spheres in hypersonic flow. We numerically investigate agglomerations of sphere pairs at a number of orientations, as well as a tetrahedral arrangement of spheres to approximate the dynamics of clustered fragments. Furthermore, free-flight experimentation in a hypersonic wind tunnel provides validation for computations and identifies potential sources of error under the present inviscid numerical modeling. The structure of the paper is as follows. In Section II, we present the numerical methodology employed and examine an exemplary test case under different levels of grid refinement. We describe the experimental apparatus and freeflight diagnostic techniques in Section III. We then detail the experimental/numerical comparison and present results from simulation surveys (Section IV) before closing with concluding remarks in Section V.

\section{Computational Modeling}

\section{A. Numerical Methodology}

As in previous studies of free-flight separation [9], we have implemented the AMROC (Automated Mesh Refinement in Object-Oriented $\mathrm{C}++$ ) [10] fluid solver to numerically compute the flows governing the separation dynamics of clustered spheres. AMROC solves the Euler equations:

$$
\partial_{t} \rho+\nabla \cdot(\rho \vec{u})=0, \quad \partial_{t}(\rho \vec{u})+\nabla \cdot(\rho \vec{u} \otimes \vec{u})+\nabla p=0, \quad \partial_{t}(\rho E)+\nabla \cdot((\rho E+p) \vec{u})=0,
$$

where $E$ represents the specific total energy and pressure is determined from the polytropic equation of state: $p=(\gamma-1)\left(\rho E-1 / 2 \mathbf{u}^{T} \mathbf{u}\right)$. All solid features are treated as embedded boundaries within a Cartesian mesh, while spatial discretization is formulated in a finite-volume flux-splitting scheme. The MUSCL-Hancock reconstruction method with a Minmod limiter is implemented by 


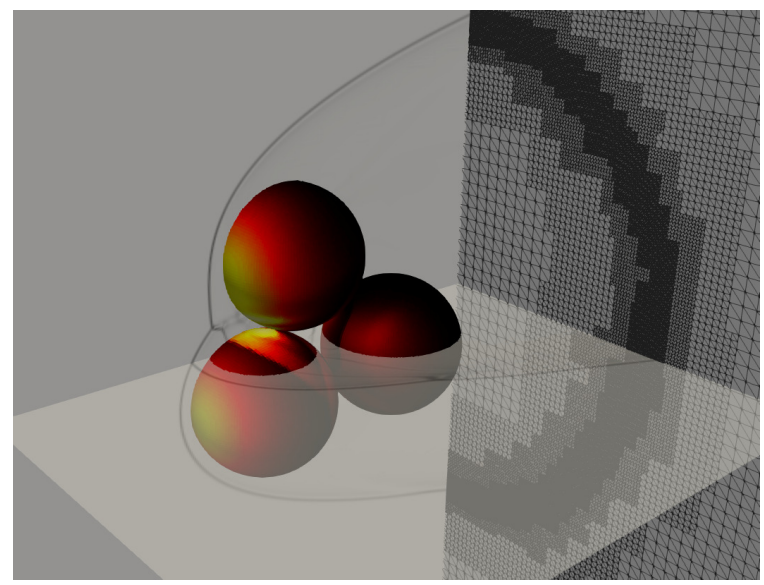

Fig. 1 Example computational mesh with three fluid refinement levels showing surface pressure, numerical schlieren, and automatically refined mesh capturing the shock.

Van Leer flux vector splitting for estimation of numerical flux at cell interfaces. Away from shocks and discontinuities, this semi-discrete formulation provides second-order accuracy, and reverts to first-order near embedded boundaries across which a ghost-fluid-based interpolation scheme mirrors primative variables. An explicit Euler time-marching scheme is conducted for all fluid simulations. Automated mesh refinement (AMR) is a critical component of AMROC that permits the effective capture of transient flow features associated with moving boundaries. The refinement process is managed by setting gradient thresholds of selected state variables and by wall proximity as determined by a level set function. The parallelized AMR method is equipped with both load-balancing and repartitioning to account for evolving mesh topologies. See Figure 1 for a representative image of AMROC's AMR capabilities.

The configuration that forms the foundation of all simulations herein is a collection of spheres placed in initial contact to approximate the physics of a fragmenting body. Consequently, mechanical contact between spheres must be modeled to allow for simulation of the initial breakup, as well as subsequent collisions between spheres. To achieve this, AMROC has been coupled with the DYNA3D structural solver [11]. DYNA3D is an explicit, nonlinear finite-element code commonly used to capture high-speed structural phenomena, and allows for a range of material models and contact physics [12]. Coupling the fluid and structural solvers is accomplished by transmission of pressure boundary conditions to DYNA3D and updated geometric boundaries to AMROC [13], along with computation of the level-set function by applying the Closest Point Transform of Mauch [14] to the triangulated surface mesh. As the base element of DYNA3D is an eight-node hexahedral, we utilize three-dimensional butterfly meshes to produce high-quality solid grids for the spherical bodies. In this study, DYNA3D is run on a single processor, while AMROC is parallelized across a larger number of processors. Boundary-condition transmission between solvers is updated serially, which, in comparison to the available parallel execution implementation, increases computational cost but ensures a higher degree of numerical stability [11].

In this work, we consider two general test cases: an arrangement in which two spheres are positioned at different alignment angles and one in which four spheres are placed at the vertices of a regular tetrahedron. Freestream conditions for both configurations were chosen to achieve a Mach number of 20, although, under the Mach number independence principle for a perfect gas, higher Mach numbers would be expected to produce nearly identical results. The density of freestream flow was set to $1 / 10,000$ of that selected for the spheres $\left(8 \times 10^{3} \mathrm{~kg} / \mathrm{m}^{3}\right.$ to approximate iron meteors), and the freestream temperature was constrained by choice of velocity $(15,000 \mathrm{~km} / \mathrm{s})$ and Mach number. Given that AMROC solves the Euler equations under a perfect-gas equation of state, the unphysically high freestream temperatures and densities exhibited here do not cause the sphere dynamics to deviate from standard inviscid flow. Note that the primary influence of real-gas effects in this setting would be to alter the shock stand-off distance near stagnation streamlines and would not be expected to modify separation trends appreciably. We employ a fully elastic material model 
for the spheres with a Young's modulus of $200 \mathrm{GPa}$ (again matching that of iron). Each sphere mesh contains 2400 surface elements, and the refinement levels of the solid and fluid domains are matched such that the maximum stable time-step size in DYNA3D roughly equates to a CFL of 0.8 in AMROC. The two-sphere simulations were conducted with spheres of $0.1 \mathrm{~m}$ radius on a $126 \mathrm{x} 50 \mathrm{x}$ 50 mesh with a computational domain of size $2.5 \mathrm{~m} \times 1.0 \mathrm{~m} \times 1.0 \mathrm{~m}$, while the tetrahedral simulations were computed on a $106 \times 64 \times 64$ mesh measuring $2.0 \mathrm{~m} \times 1.2 \mathrm{~m} \times 1.2 \mathrm{~m}$. On the highest refinement level, a sphere is spanned by $\sim 40$ fluid cells. Two additional levels with refinement factors of two were allowed based on density-gradient threshold and wall proximity criteria. During a simulation, a period of $3 \mathrm{~ms}$ is allotted to establish quasi-steady flow over the stationary spheres; at this point, they are allowed to fly freely for $8 \mathrm{~ms}$ until program termination, which was deemed sufficiently long based on nondimensional interaction times presented in other studies $[8,9]$. However, in certain two-sphere cases, we allow the simulation to run for an additional $4 \mathrm{~ms}$. Typical computational times on a Dell Precision T7820 were $~ 800$ CPUh and 1,300 CPUh for the two- and four-sphere simulations, respectively.

\section{B. Model Verification}

To assess the computational reliability of the present numerical model, we perform a limited grid-refinement study of sphere separation from a chosen initial arrangement. The scenario of interest here considers four spheres in a tetrahedral arrangement with flow parameters as described in Section II A. The chosen geometry exhibits several behaviors characteristic of four-sphere separation and, therefore, was deemed a suitable case for verifying the convergence of the coupled fluid/solid solver. The trajectory details of this particular case can be found in Section IV C, with representative images provided in Figure 12. The verification study consists of two simulations, each with spheres of $0.1 \mathrm{~m}$ radius on a $106 \times 64 \times 64$ base mesh measuring $2.0 \mathrm{~m} \times 1.2 \mathrm{~m} \times 1.2 \mathrm{~m}$. A coarse simulation utilized two levels of refinement, while a fine mesh included a third level of refinement. The solid meshes employed in each case were constructed to match the size of the finest fluid cells, and timestep sizes were automatically adjusted to match a CFL condition of 0.8 . Both computations were run on a 56-core Dell Precision T7820 workstation; the coarse simulation required $\sim 1,300$ CPUh of computation to complete, while the fine simulation, at $\sim 18,000$ CPUh, was significantly more expensive.

In Figure 2, we present differences in sphere positions and lateral velocities between the coarse and fine simulations, finding that no coarse sphere deviates from its fine position by more than $r_{\mathrm{sph}} / 2$ over the simulation duration. The differences exhibited by spheres 1, 2, and 3 through $2 \mathrm{~ms}$ indicate disparities in the initial impulses experienced, which is consistent with minor qualitative differences in the initial surface pressure distributions shown in Figure 3. As the simulation proceeds, spheres 1, 3 , and 4 seem to accrue only modest positional errors (notwithstanding persistent collisions between spheres 1 and 4), while it is apparent from the diverging motions of sphere 2 that begins to follow a different trajectory class altogether. In the coarse simulation, sphere 2 transitions from shock-surfing to entrainment in the wake of sphere 1, while fine sphere 2 continues to surf the shock of sphere 1 until it exits the domain. These observations are in agreement with differences in lateral velocity, which is a primary quantity of interest in studies of free-flight separation. The variation of spheres 1,3 , and 4 is limited to $\sim 5 \%$ of the final lateral velocity, suggesting that the general separation behavior of these bodies is consistent in both coarse and fine simulations. As with its positional deviation, coarse sphere 2 begins to diverge from its fine lateral velocity at $\sim 2 \mathrm{~ms}$, following a disparate course through the computational domain. The cause for such a severe discrepancy likely results from a combination of the high sensitivity of shock-surfing trajectories to initial conditions and the inability of the coarse simulation to resolve the complex set of swept shock-shock interactions and shock reflections dictating the surface pressures on spheres 1 and 2. Indeed, examination of lateral force coefficients in the fine simulation shows a $\sim 7 \%$ increase in the repulsive force on sphere 2 at the moment of release. Despite difficulties accurately capturing the motion of sphere 2, the coarse simulation manages both to resolve the shock-shock interaction dictating the expulsion of sphere 3 and to reproduce the lateral velocities of spheres 1 and 4 after a prolonged series of collisions. In spite of the discrepancies exhibited in the trajectory of sphere 2 in this refinement study, we find that the numerical model is capable of achieving valid general behaviors of spheres in the coarse simulation, justifying our use of the coarse mesh parameters through the rest of this work. 


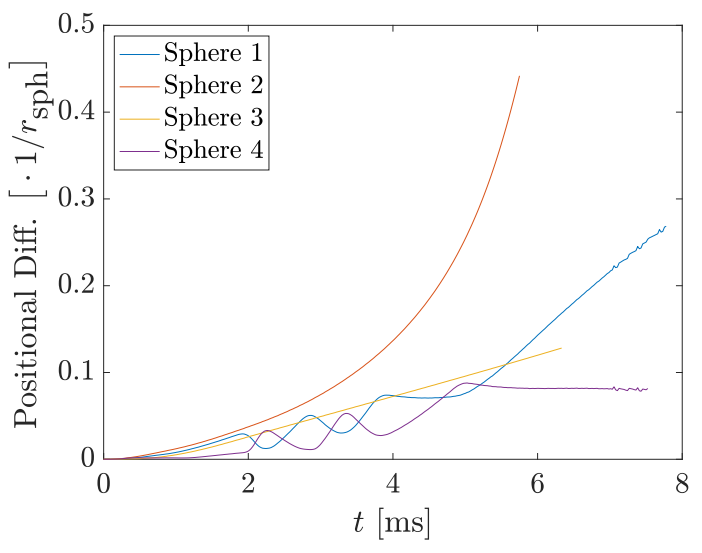

(a)

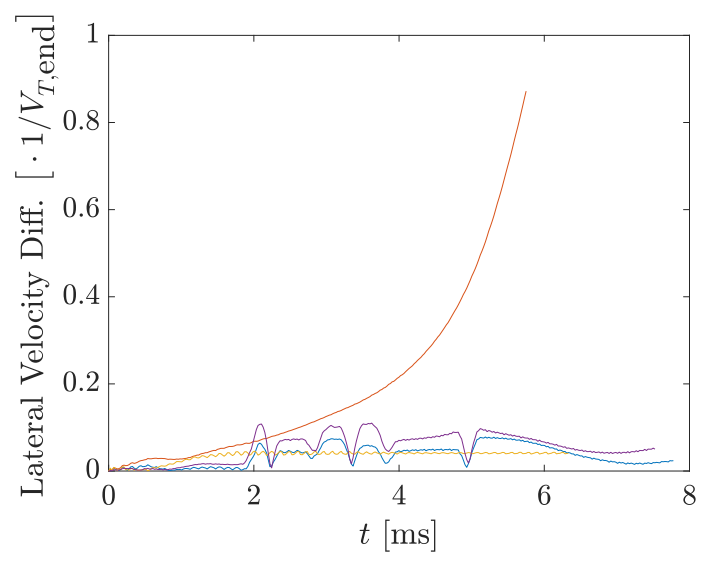

(b)

Fig. 2 Difference between sphere (a) position and (b) lateral velocity in coarse and fine simulations.

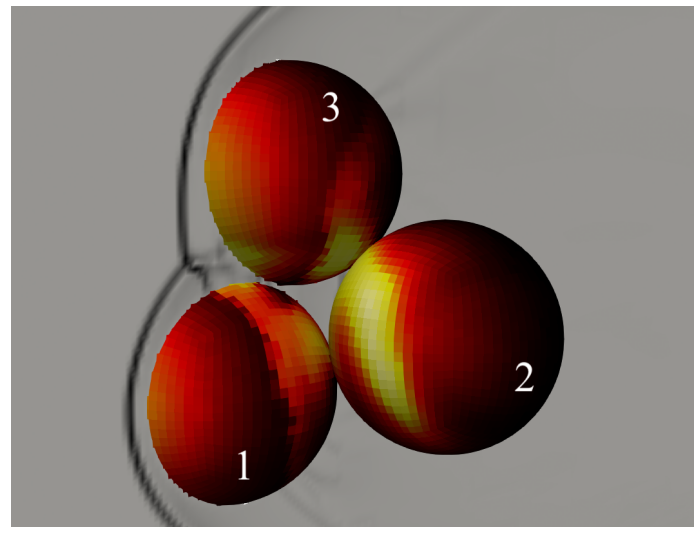

(a)

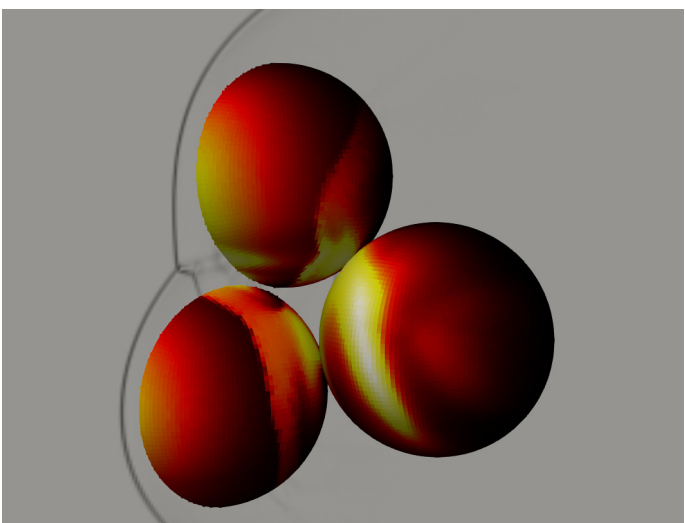

(b)

Fig. 3 (a) Coarse and (b) fine centerline pseudo-schlieren with surface pressure at sphere release from model verification simulations.

\section{Experimental Apparatus}

\section{A. Facility}

The experimental facility employed in this study was the Hypersonic Tunnel for Educational and Research Purposes (HyperTERP) at the University of Maryland [15]. HyperTERP is a reflected shock tunnel equipped with an axisymmetric contoured nozzle with a $220 \mathrm{~mm}$ exit diameter providing a nominal Mach number of 6 . The test section of the tunnel measures $305 \mathrm{~mm}$ in diameter, with optical access provided by $152 \mathrm{~mm}$ diameter windows on the top, bottom, and sides. The driver and driven sections of the tunnel measure $5.4 \mathrm{~m}$ and $10 \mathrm{~m}$ long, respectively, each with an inner diameter of $100 \mathrm{~mm}$ (a labeled tunnel schematic is provided in Figure 4). To operate the tunnel, the entire facility is vacated to near-vacuum conditions, at which point the driven section of the upstream shock tube is filled with a dried air test gas. The driven section is pumped to high-pressure conditions with a mixture of dried air and helium; the exact pressure is dictated by the shock-tube tailoring conditions needed to extend the nominal steady test time. A standard double-diaphragm is utilized to provide precise control over test initiation. The ensuing shock advances towards a secondary diaphragm located immediately upstream of the nozzle; this thinner mylar sheet ruptures upon shock reflection, allowing test gas from the high-pressure and -temperature reservoir to flow through the nozzle and begin the test. Reservoir conditions are estimated on a run-to-run basis by measuring the propagation speeds of the incident and reflected shocks using high-speed $\mathrm{PCB}^{\mathrm{TM}}$ pressure transducers mounted in the shock-tube walls. The steady test time concludes when the 


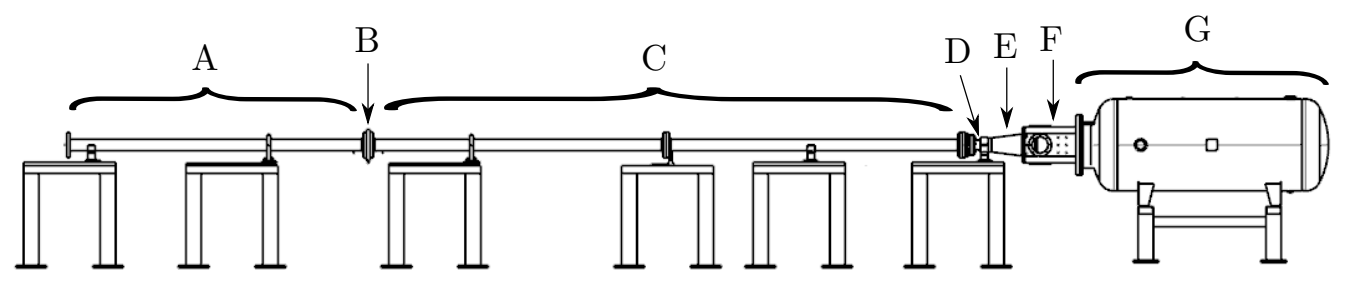

Fig. 4 Schematic of HyperTERP hypersonic wind tunnel with (A) driver section, (B) doublediaphragm, (C) driven section, (D) secondary diaphragm, (E) Mach 6 nozzle, (F) test section, and (G) dump tank.

expansion from the primary diaphragm rupture reaches the reservoir after reflecting off the opposite end of the shock-tube. The facility can be operated at arbitrary reservoir conditions, but we utilize only those for which proper tailoring has been achieved. In this experimental campaign, we employed a test condition which yielded a stagnation pressure of $1.33 \mathrm{MPa}$ and stagnation temperature of $921 \mathrm{~K}$, equating to freestream conditions of $847 \mathrm{~Pa}, 112 \mathrm{~K}$, and $1275 \mathrm{~m} / \mathrm{s}$ at a Mach number of 6. Experimental steady test times for this operating condition were generally limited to $10 \mathrm{~ms}$.

\section{B. Free-Flight Models}

All experiments in this campaign utilized spheres as test models. In particular, we tested clustered spheres of diameter $6.35 \mathrm{~mm}$ in a tetrahedral configuration. The spheres used were made from Delrin ${ }^{\circledR}$ Acetal plastic of $<\% 1$ deviation from sphericity, and, with a density of $1,411 \mathrm{~kg} / \mathrm{m}^{3}$, they typically traverse much of the visible region of the test section during the steady flow time. The principal difficulty of free-flight experimentation is keeping the test model suspended in the correct orientation before the test begins and allowing it to move uninhibited thereafter. Previous studies have glued frayed dental floss to free-flight models with reasonable success [9]; for larger numbers of spheres, however, this methodology is not feasible. Instead, we rely on three-dimensional printing methods to hold the spheres in a desired position. Given a set of sphere locations, a typical 3Dprinted suspension model will consist of two circumscribing hemispherical shells which are molded to force the test spheres into a particular orientation. The supporting hemispheres are additionally fitted with center-of-mass-aligned channels for suspension tethers, slightly misaligned dowel/hole pairs to keep the models together, and a set of upstream and downstream tabs to promote separation upon flow arrival (see Figure 5 for a representative model). The large upstream flaps are angled $30^{\circ}$ to the freestream and are printed as frames which are then covered with Kapton tape to reduce the shell mass. Frayed dental floss holds the entire formation from the test-section roof; the floss breaks when the tunnel starts, imparting negligible impulse to the model and allowing the 3D-printed model to separate solely by aerodynamic means. The circumscribing shells thus must be lightweight to ensure that they break apart from the initial configuration quickly, leaving the interior spheres undisturbed in the freestream. The size of the mold varies with sphere configuration, but a shell/flap thickness of $0.75 \mathrm{~mm}$ regularly results in separation times on the order of $1.5 \mathrm{~ms}$. All 3D-printed models are fabricated by a Formlabs Form 2 stereolithography printer, which, with a layer thickness of $50 \mu \mathrm{m}$, is capable of resolving fine model details. The mildly adhesive finish of the resin-based models is detrimental to facilitating a smooth breakup process, so all shells and spheres are coated with a thin layer of talcum powder to mitigate disturbance of the test configuration.

\section{Stereoscopic Tracking}

Tracking of sphere motions is achieved entirely by nonintrusive optical methods. A two-camera setup with sufficient angular separation is required to reconstruct the three-dimensional positions from projected visualizations. A shadowgraph arrangement is utilized on the horizontal imaging plane; essentially a schlieren with knife-edge removed, the shadowgraph captures the sphere outlines and weakly visualizes flow features to elucidate the aerodynamics without compromising tracking accuracy. A Vision Research Phantom v2512 operated at $32 \mathrm{kHz}$ is paired to a Cavitar Cavilux HF laser for illumination. The second camera view uses standard imaging from below the test section, again with a Phantom v2512 and driven at the same frame rate as the first. Back-lighting is provided by an LED with several layers of diffuser sheeting, which produces a uniform imaging background. 


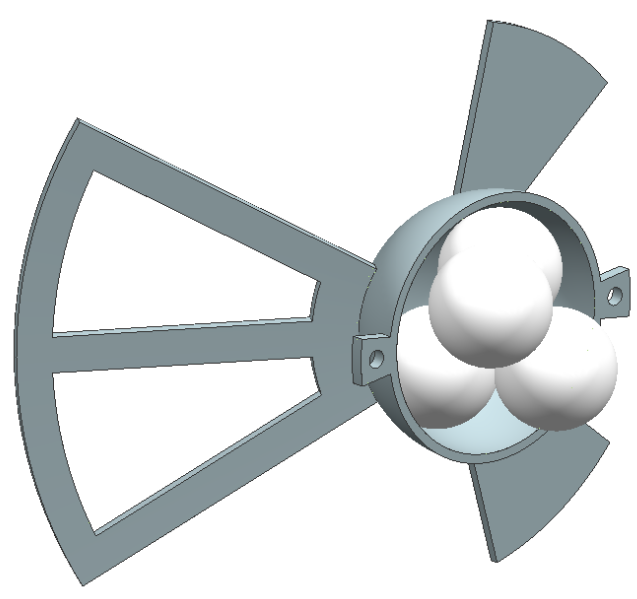

(a)

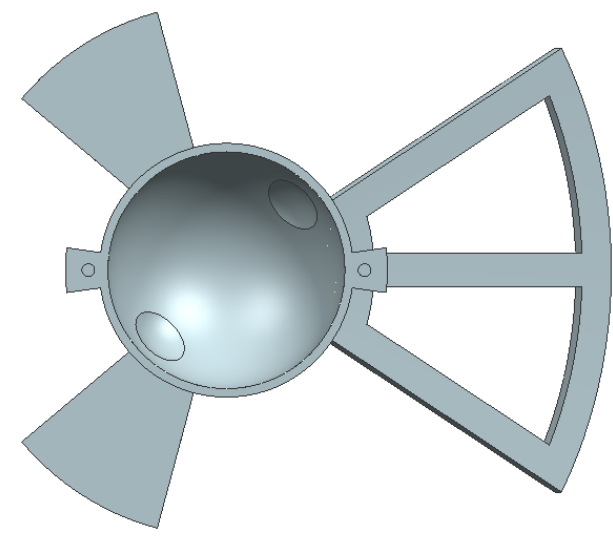

(b)

Fig. 5 (a) Computer-animated hemispherical shell holding spheres in a tetrahedral formation and (b) side view of opposing shell showing molded spherical caps.

Prior to camera calibration, tracking of free-flight bodies is performed using edge-detection routines from Laurence and Karl [16], which employ Canny edge-detection of a sphere silhouette and sub-pixel edge tracing to perform a linear least-squares fit of its location in pixel coordinates. Overlap between bodies is inevitable in multi-sphere testing environments, so we use a semi-automated routine to determine the proper limits of a detected edge. Calibration of the camera apparatus relies on the assumption of two distinct camera models; the vertical view is described by the pinhole model and the horizontal shadowgraph by orthographic projection. For the purposes of calibration, we place the pinhole camera at the origin and, due to the lack of depth information in orthographic projection, constrain the shadowgraph camera to lie at a position $1 \mathrm{~m}$ away from the test section. Next, we fix the internal camera parameters (i.e., scale and projective center) by imaging a $9 \times 16$ grid of markers on a hand-held plate at various orientations. Calibration of the relative camera positions and orientations is then performed in-situ by adhering to the projection laws governing each camera model and using a bundle-adjustment routine to minimize the reprojection error of a subset of tracked sphere positions. Finally, we reconstruct three-dimensional sphere positions for the full test by solving for the points of minimum distance from the skew projective lines. In general, the camera are separated by $90^{\circ}$, but the use of a tripod system sensitive to minor disturbances necessitates the run-to-run self-calibration technique. Uncertainties in the reconstructed three-dimensional positions are estimated to a characteristic value of $0.0302 \mathrm{~mm}$, or $\sim 0.01 r_{\mathrm{sph}}$, based on the mean reprojection error over the test duration. A schematic of the stereoscopic camera setup is provided in Figure 6 .

\section{Results and Discussion}

\section{A. Comparison of Experimental and Computational Results}

To provide a basis for the validity of the coupled numerical methodology, we present a comparative assessment of a selected experiment of separating free-flight spheres and a corresponding simulation with fully matched conditions. The chosen experiment features four spheres of diameter $6.35 \mathrm{~mm}$ arranged into a tetrahedron (as in Section IVC), with two leading spheres at approximately similar streamwise locations and a pair of roughly aligned trailing spheres. Simulations of spheres at a similar orientations reveal that this arrangement is expected to produce extended shock surfing, a scenario highly sensitive to relative sphere positions and a sensible benchmark test of the resultant computational aerodynamics. In Figure 7, a sequence of images taken from below and from the side of the test section depict the sphere trajectories, along with a three-dimensional reconstruction of the extracted positions, where time zero here is the point at which the counterpart numerical simulation draws its initial kinematics. Shocks, though faint, are visible in the central column, which helps provide some degree of information about the underlying fluid dynamics. As shown in the $t=1.40 \mathrm{~ms}$ frame, the two leading spheres experience strong repulsive forces and 


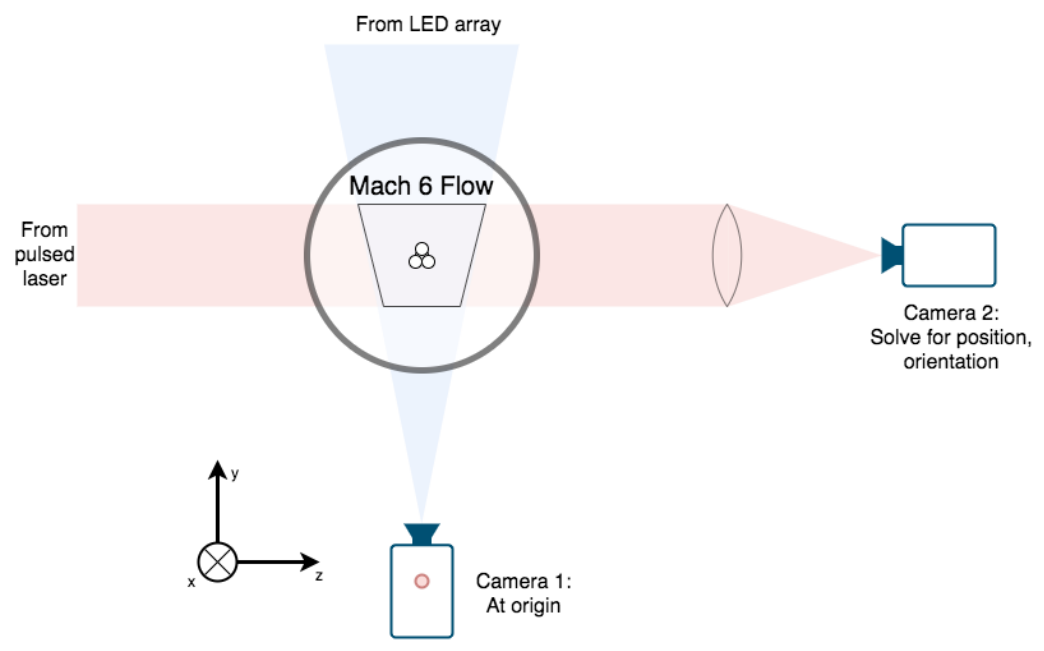

Fig. 6 Schematic of stereoscopic camera setup.

almost immediately separate laterally, at which point the trailing spheres are no longer shielded from the freestream flow. Indeed, in the shadowgraph visualization of $t=2.80 \mathrm{~ms}$, impingement of the shock from sphere 1 on sphere 4 is apparent and the resulting drag augmentation is reflected in the increased positional separation between the two. Note that sphere 3 likely also receives the bow shock from sphere 1 , but the associated flow features are not visible because the density disturbance is far removed from its tangency point with the pulsed beam. In any case, it appears that spheres 1 and 2 have ceased aerodynamic interaction by frame $t=2.80 \mathrm{~ms}$, while 3 and 4 remain under the influence of sphere 1 for an extended period of time. From the three-dimensional reconstruction, sphere 3 has begun to fall into the wake of sphere 1, while the shock-shock interaction on sphere 4 moves further towards its inboard side (see central frames of $t=4.20-7.00 \mathrm{~ms}$ ), indicating an eventual expulsion trajectory. The trailing spheres have left the stereoscopic viewing volume by $t=7.00 \mathrm{~ms}$, and the leading spheres continue on undisturbed trajectories until completion of the experiment.

The complementary simulation exhibits qualitatively similar behavior, as evidenced in the projected numerical sphere positions in the left and center columns of Figure 7. While we do commence sphere tracking at $t=-0.375 \mathrm{~ms}$ in the experiment, the suspension shells have not yet separated far enough from the spheres. Instead, at the selected $t_{0}$, the cluster has established its own bow shock and the sphere dynamics are unlikely to be influenced much by the shells, so any discrepancy between simulation and experiment is considered to be independent of the suspension/release mechanism. Note also that the spheres possess nonzero initial velocities resulting both from contact forces as the shells break apart and from pressure forces before the spheres achieve aerodynamic independence from the shells. These initial velocities are limited to $0.0015 u_{\infty}$ and are achieved in the simulation by applying brief impulses to the spheres synchronous with the commencement of the coupled portion of the computation. As a quantitative measure of the agreement between experiment and simulation, we present a comparison of positional errors relative to the sphere radius in Figure 8a and lateral velocity errors scaled by the final experimental value in Figure 8b. Most spheres follow the same general paths in both experiment and simulation, although sphere 4 shows significant deviation beginning at roughly $t=4 \mathrm{~ms}$. Relative to the experiment, this sphere exhibits reduced displacement in the streamwise direction, while the lateral motion follows that of sphere 1, indicating that it is beginning to follow a wake-entrainment trajectory (in agreement with visualization of computational flowfield results). The other major discrepancy in the results of the numerical modeling is the streamwise positions of the leading spheres. As is expected in a simulation that neglects viscosity, the drag forces on the spheres, particularly those exposed to unimpeded freestream flow, will be reduced compared to experimental results. Despite the positional differences of the other bodies and the complex interaction to which it is subjected, sphere 3 exhibits very low deviation from its experimental trajectory. Also, spheres 1, 2, and 3 accrue positional errors as the simulation progresses, but their lateral velocities remain fairly true to the experimental results, as 

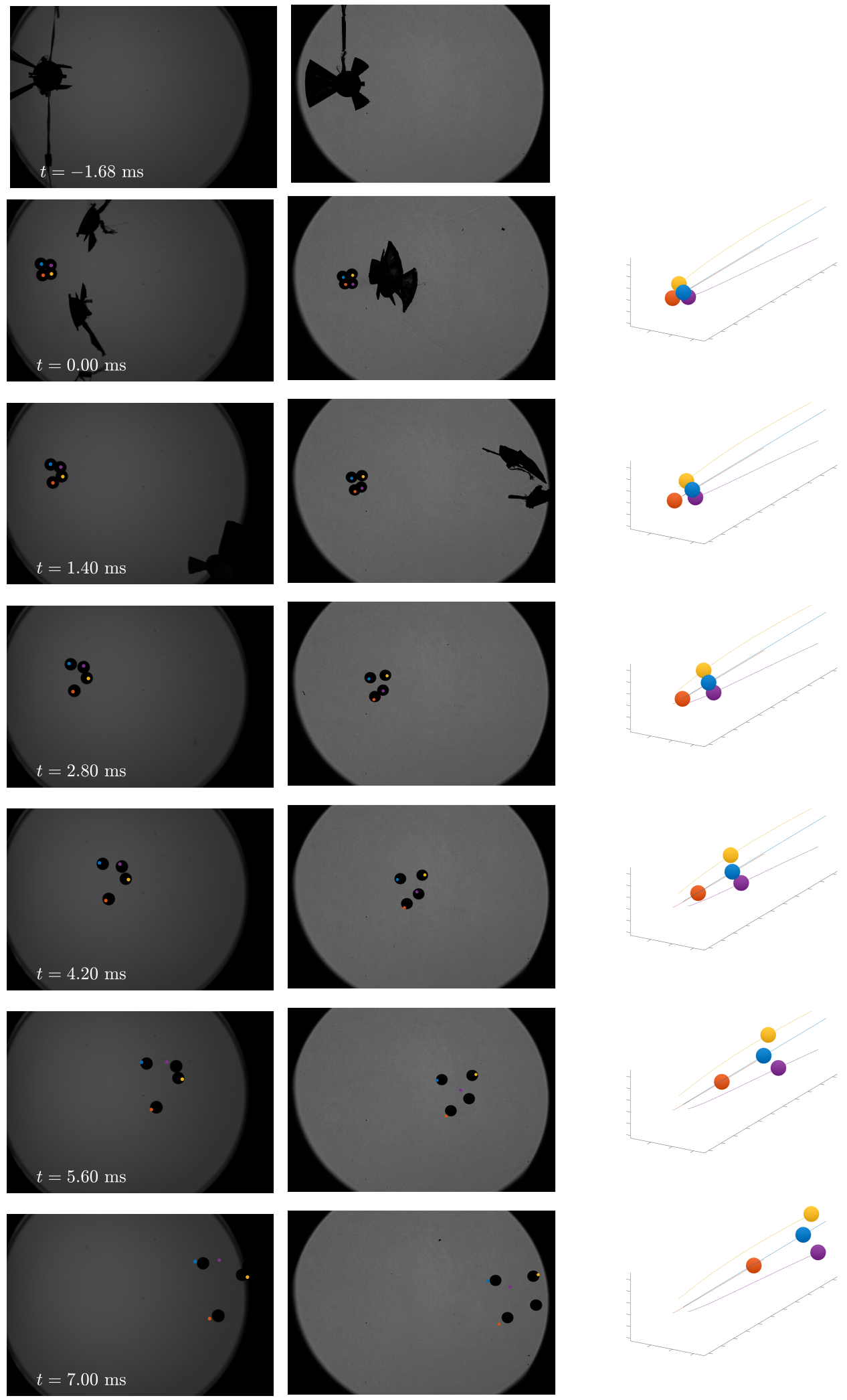

Fig. 7 Separation sequence of four-sphere validation experiment from (left) bottom standard camera, (center) side shadowgraph camera, and (right) positional reconstruction with the following color scheme: (blue) sphere 1, (red) sphere 2, (yellow) sphere 3, (purple) sphere 4. Markers in the left and center columns indicate numerical sphere positions. 


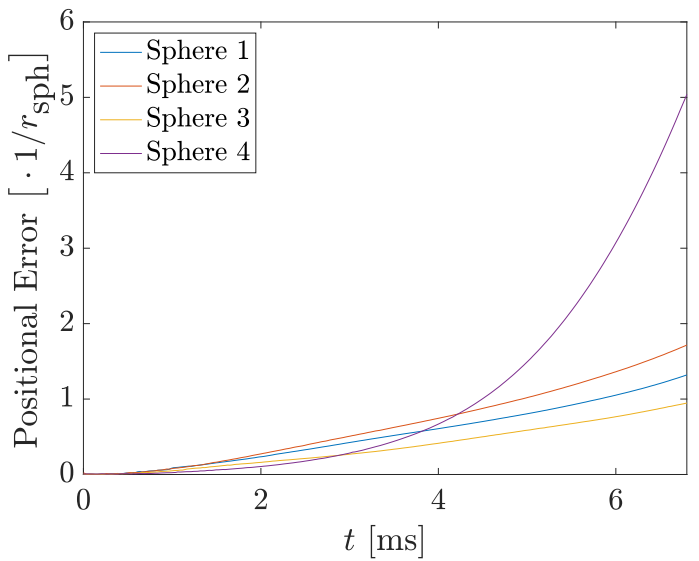

(a)

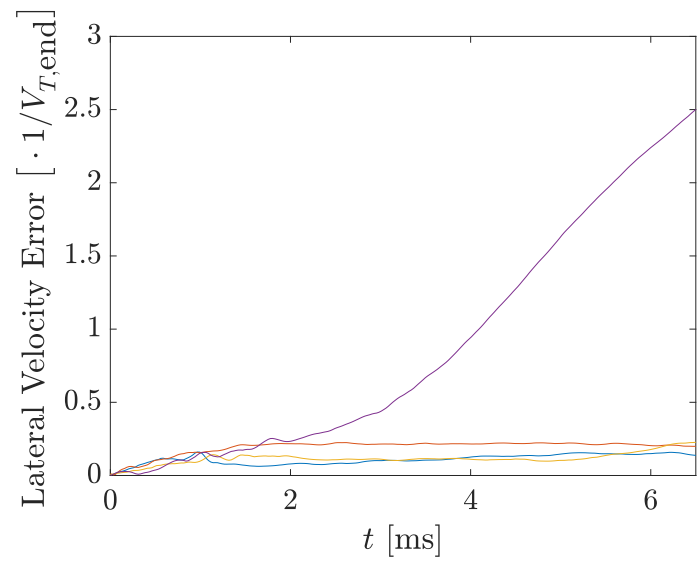

(b)

Fig. 8 (a) Positional error between computation and experiment relative to sphere radius and (b) error in lateral velocity relative to value at end of experiment.

errors are limited to only $\sim 20 \%$ of their respective final values.

The discrepancy between the experimental and computational trajectories is likely to stem directly from the difference in the nominal drag coefficient for viscous and inviscid spheres. In the numerical interaction between spheres 1 and 4 , the streamwise distance between bodies is greater due to the reduced drag on the primary. As a result, the bow shock has spread further laterally at the streamwise position of the secondary sphere and impinges further towards its outboard flank, which, in turn, reduces the effective lift. When the lift-to-drag ratio of the secondary body falls below the tangent of the local shock angle, it will become entrained in the wake of the primary, which is evidently what occurs in the simulation of interest. Another plausibly contributing factor is that the moderately high enthaply freestream flow could activate vibrational relaxation along streamlines passing through strong shocks in the flowfield, thereby reducing the shock stand-off distance and possibly translating the outer portions of the shock inwards. Based on the trajectory discrepancy of sphere 4 in this particular scenario, we might expect a systematic underestimate of sphere separation velocities under the current numerical methodology. Nonetheless, the other bodies remain within one sphere diameter of their experimental positions for the entire simulation and exhibit low deviation in lateral velocity, so we deem the numerical model of coupled AMROC/DYNA3D well-suited for reproducing the general behaviors exhibited by spheres separating from clusters.

\section{B. Two-Sphere Separation}

We begin by examining the separation of the simplest possible arrangement: two initiallytouching spheres at various alignment angles. The aerodynamics of this scenario have been well characterized in previous studies, but the effect of surface contact has not yet been investigated. In particular, the terminal behavior of spheres arranged into a streamwise-aligned formation (i.e., no immediate separation) is still unknown despite the relatively fundamental nature of the problem. A survey of spheres at different alignment angles will help elucidate some of the physics influencing the dynamics in this regime. In this section, we adopt a convention wherein $\theta$ represents the alignment angle between the center of the leading sphere and that of the trailing, with an angle of $180^{\circ}$ indicating that the secondary sphere is directly behind the primary. We have thus simulated the trajectories of spheres at alignment angles of $90^{\circ}$ to $180^{\circ}$ in increments of $7.5^{\circ}$. As an example of a typical separation sequence, we provide a visualization of the simulation at $\theta_{0}=172.5^{\circ}$ in Figure 9 . Here the spheres are closely aligned with the freestream flow, resulting in negligible aerodynamic forces on the trailing sphere and significant contact forces between the pair. Maintaining surface contact, the spheres then seem to rotate about each other towards lower $\theta$ values until repulsive forces on the trailing sphere trigger separation. The resulting trajectorial behavior is thus similar to a scenario in which the spheres are immediately repelled.

We now present in Figure 10a the trajectories of all secondary (trailing) spheres in the polar 

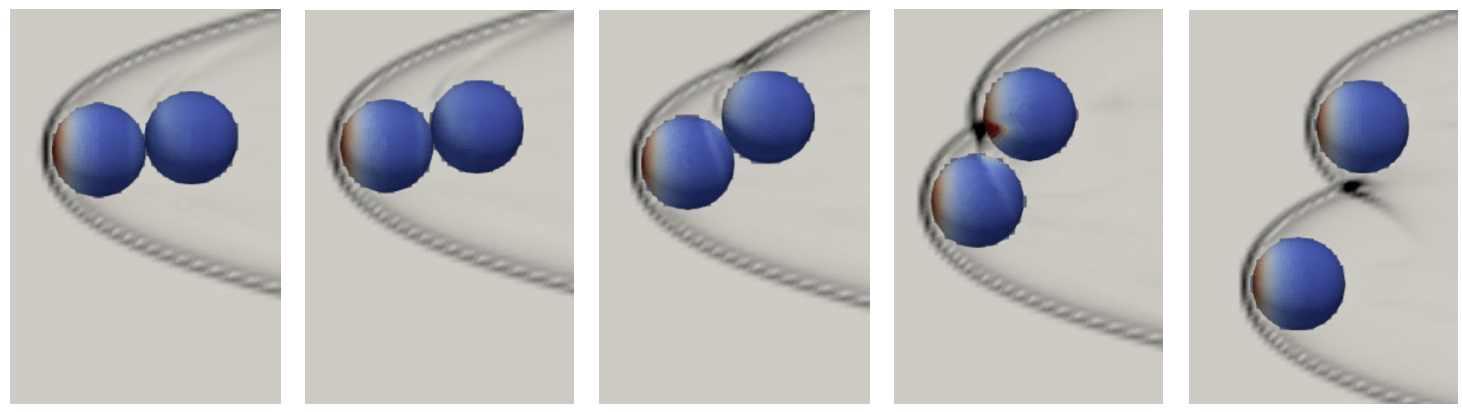

Fig. 9 Example separation procedure for two spheres initially aligned at $172.5^{\circ}$. Computational schlieren visualized along centerline with surface pressure coloring on spheres. Images shown in steps of $t^{\prime}=4.2$.

coordinate system of the primary (where the radial coordinate represents the edge-to-edge separation of the spheres), as well as lateral sphere velocities from the system's center of mass in Figure 10b under the following nondimensionalizations:

$$
\begin{aligned}
V_{T}^{\prime} & =\sqrt{\frac{\rho_{\mathrm{sph}}}{\rho_{\infty}}} \frac{V_{T}}{u_{\infty}}, \\
t^{\prime} & =\sqrt{\frac{\rho_{\infty}}{\rho_{\mathrm{sph}}}} \frac{u_{\infty} t}{r_{\mathrm{sph}}} .
\end{aligned}
$$

The behavior near alignment angles of $\theta_{0}=90^{\circ}$ closely conform to expectations from previous studies: the spheres immediately separate under the influence of high inboard pressures near the stagnation region of the spheres and achieve a nondimensional separation velocity of 0.2 . As the secondary is shifted further back in the formation (up to $120^{\circ}$ ), it undergoes some degree of shock surfing, which increases its terminal transverse velocity $(\sim 0.25)$. At $127.5^{\circ}$, the secondary sphere exhibits brief surfing before becoming entrained in the wake of and colliding with the leading sphere; however, the simulation was terminated before the eventual trajectories of the spheres were made clear. Spheres at $135^{\circ}$ and $143^{\circ}$, on the other hand, remain in contact for the duration of the simulation; examination of surface pressures on the trailing sphere reveals that, as the alignment angle decreases, a restorative force drives the arrangement back toward larger $\theta$ values. As the initial orientation is rotated further towards alignment with the freestream, the trailing sphere tends to 'roll' along the surface of the primary until surface contact is lost and the bodies separate in a similar manner to the lower $\theta_{0}$ scenarios. At $180^{\circ}$, no discernible change in alignment angle was recorded, indicating the presence of an equilibrium position. Noticeably absent in Figure 10a are secondary spheres that persist in the wake of the primary, which seems to signal only three ultimate trajectories: immediate separation, delayed separation, and indefinite contact. Indeed, Figure 10b reflects this quasi-bimodal behavior with two distinct sets of final lateral veolcities. The cases exhibiting both immediate and delayed separation tend towards nondimensional velocities of $\sim 0.2$, while persistent contact trajectories display near-zero velocity oscillations resulting from the elastic nature of the bodies.

To gain further insight into the apparent stability behavior of the sphere pair at $\theta_{0}=135^{\circ}$ and $142.5^{\circ}$, we model the motions of both spheres as a single rigid body (that is, while relative forces along the contact line are greater than zero) and extract from the simulations a database of forces and moments for use in applying dynamics to the two-sphere system. Based on the computed forces on each sphere, the bodies will remain in contact for polar angles above $132^{\circ}$, and thus all trajectories simulated under the rigid-body assumption must remain above this limit. Comparison of the simplified model with computational trajectories in phase space show qualitatively similar behavior (see Figure 11). It should be noted, however, that, while the comparative positional coordinates follow nearly the same trajectories, the computational spheres move much more quickly (possibly due to sliding motion), so we have scaled $\dot{\theta}_{C F D}$ by a factor of $1 / 6$ to roughly match the ordinate limits of the modeled spheres. Nevertheless, with this empirical scaling, rigid-body modeling shows reasonable agreement with the simulations and can thus be used to estimate the range of kinematics allowing for contact stability. The bounding loop of stability (shown in gray) is 


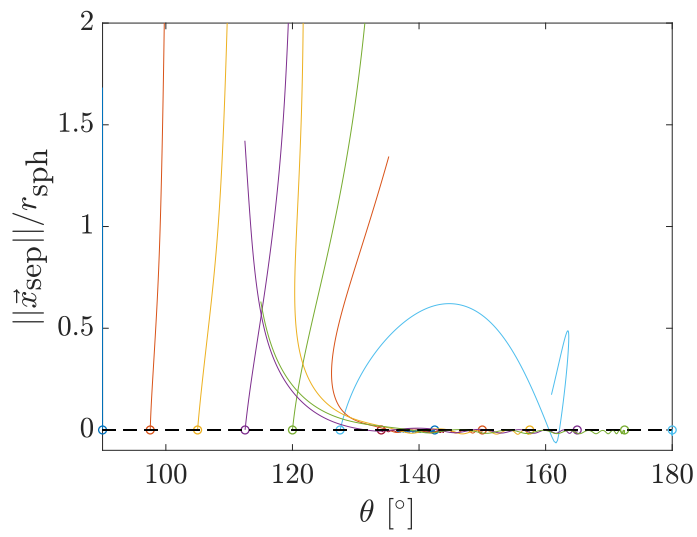

(a)

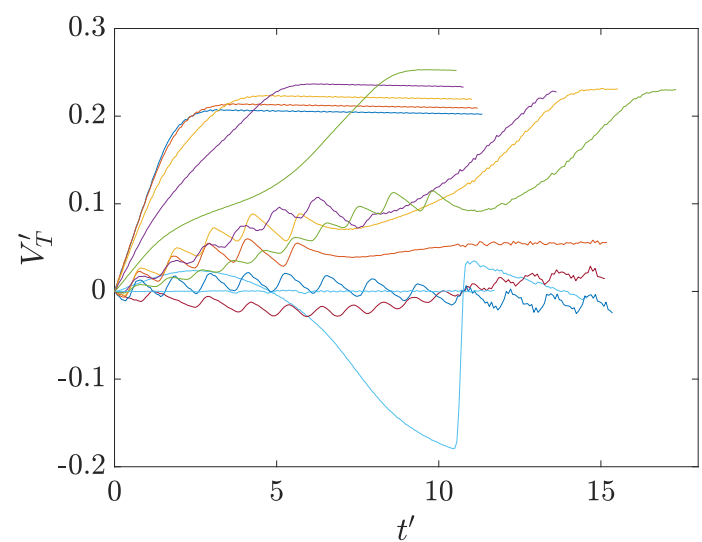

(b)

Fig. 10 (a) Polar trajectory map and (b) nondimensional lateral velocity of two-sphere survey.

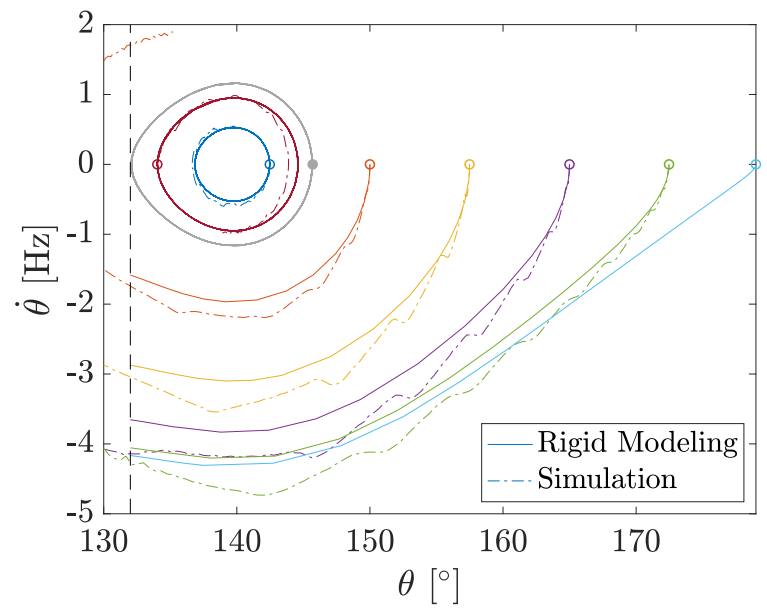

Fig. 11 Phase space behavior of spheres that initially remain in contact.

contained by initial alignment angles of $132^{\circ}$ and $145.7^{\circ}$, while the maximum allowable rotational speed is $\sim 1 \mathrm{~Hz}$. Although this is a narrow stability band in highly specialized circumstances, the implications of a binary body resisting breakup during atmospheric transit are significant for energy deposition considerations, as the effective retention of inertia will allow the body to penetrate deeper into the atmosphere. Contact stability for bodies of smaller radius ratios caused by aerodynamic shielding has been noted by Register et al. [8], and its persistence to bodies of equal size suggests that a stable regime exists for a wide range of relative sphere sizes. Also, there seems to be an unstable equilibrium point located at $\theta_{0}=180^{\circ}$, but the simulation was likely too short for perturbations to drive the spheres onto a separation trajectory.

\section{Tetrahedral Separation}

While the aerodynamic separation of binary spheres is a well-studied process, it lacks the complexity of fragmentation events likely to occur during the entry of meteors. As an initial exploration of the separation characteristics of sphere clusters, we employ numerical simulations to examine a fundamental multi-sphere arrangement: four equal-sized spheres positioned such that their centers are located on the vertices of a regular tetrahedron. Assuming a standardized formation rather than a random agglomeration allows for systematic variation of the governing parameters, in this case the pitch and yaw angles of the cluster (note that separation behavior is degenerate under roll transformations). Although the definition of principal orientation is arbitrary in this setting, we adopt a convention for $0^{\circ}$-pitch $/ 0^{\circ}$-yaw in which three spheres form a streamwise-normal plane 

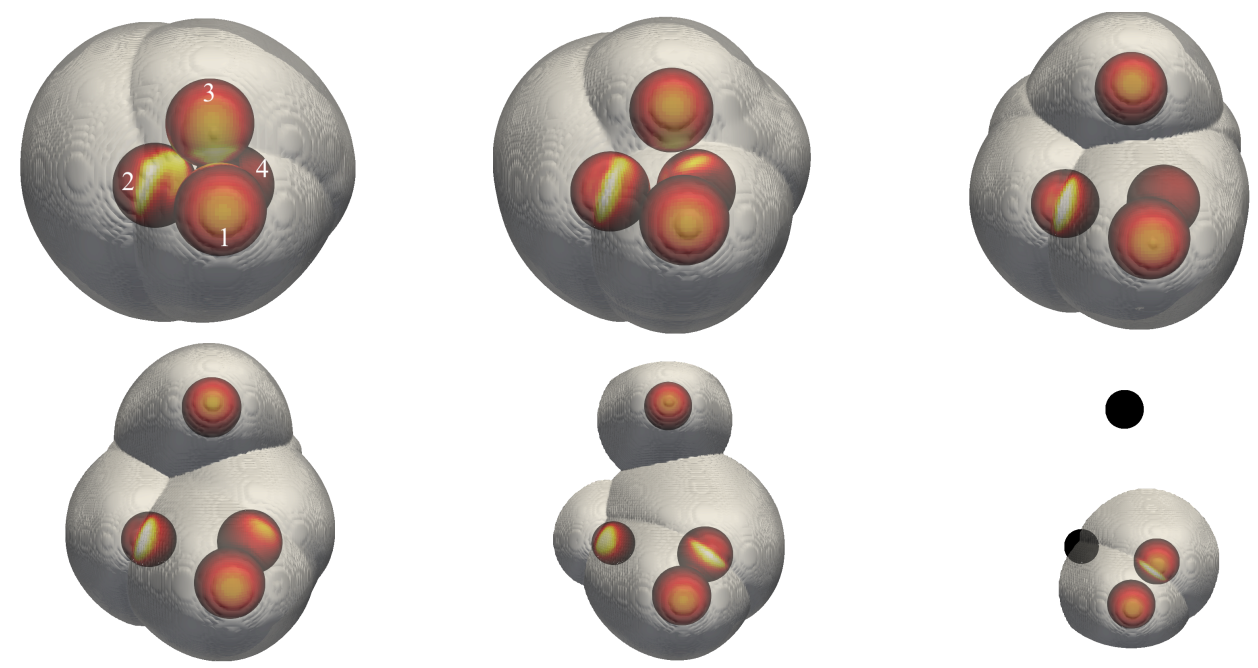

Fig. 12 Release of tetrahedral sphere cluster into Mach 20 flow with surfaces colored by pressure and primary shock structure visualized in gray. Images in steps of $t^{\prime}=2.1$.

behind a single leading sphere. The simulated parameter space spans angles of $-36^{\circ}-54^{\circ}$ in pitch and $0^{\circ}-90^{\circ}$ in yaw at intervals of $18^{\circ}$, with several additional tests performed to capture the full extent of separation behaviors. In total, 38 simulations were conducted in this configuration.

First, to highlight some of the governing dynamics in a four-body scenario, we review the features of a typical separation sequence of spheres initially placed in a tetrahedron. In Figure 12, we present a set of snapshots visualizing instantaneous sphere positions colored by surface pressure and the associated primary shock structures on the first refinement level from an initial orientation of $36^{\circ}$-pitch $/ 18^{\circ}$-yaw, as well as force coefficients in Figure 13. Note that this is the same simulation used for verification of the numerical model. In the cluster's initial state, an encompassing bow shock generated principally by sphere 1 impinges on spheres 2 and 3 , while sphere 4 is shielded in its wake. The shock-shock interaction on the inboard side of sphere 3 results in a region of high surface pressure on both spheres 1 and $3\left(C_{L} \approx 0.45\right)$, promoting immediate lateral repulsion. Consequently, sphere 3 is aerodynamically independent from the rest of the cluster by $t^{\prime}=4$. Sphere 2 resides at a location further downstream in the cluster and so is subjected to both shock impingement from sphere 1 and the swept shock-shock interaction between spheres 1 and 3. Both occur towards the front of the body, which induces augmented drag and increases the initial impulse in the streamwise direction. As in the visualization of Figure 12, sphere 3 remains in a shock surfing trajectory for an extended period of time before beginning to follow an entrainment trajectory in the wake of sphere 1 . On the other hand, sphere 4, located firmly in the wake of the leading sphere, experiences highly reduced drag which initiates a prolonged series of collisions between the two before 'rolling' away from sphere 1, as in Section IV B. Due to the delayed onset of repulsive forces on sphere 4, it trails sphere 1 laterally and eventually exhibits separation further towards the cluster center than sphere 1. While the spheres have not all reached their final aerodynamic state before exiting the computational domain, we find this simulation illustrative of the variety of behaviors observed in the survey.

The nondimensional separation coefficient, $C$, a common metric for understanding the degree of repulsion in a given scenario, refers to the distance between two bodies when lateral forces vanish, and, in practice, it is used as a scaling factor for the dimensional separation velocity. However, Laurence et al. [5] showed that lateral edge-to-edge distance is not a valid indicator for the presence of aerodynamic interference for two bodies, as is especially clear in the case of extended shock surfing. Furthermore, in the context of more than two bodies separating, this criteria is ill-defined, as a primary reference body may not exist in a given cluster and repulsion between fragments does not necessarily cease simultaneously. We accordingly find it essential to redefine both the lateral separation velocity and separation coefficient $C$ to ensure applicability for scenarios with an arbitrary number of bodies. We note, as above, that lateral momentum is clearly not conserved in a system of aerodynamically interacting bodies; therefore, a critical quantity in assessing the separation charac- 


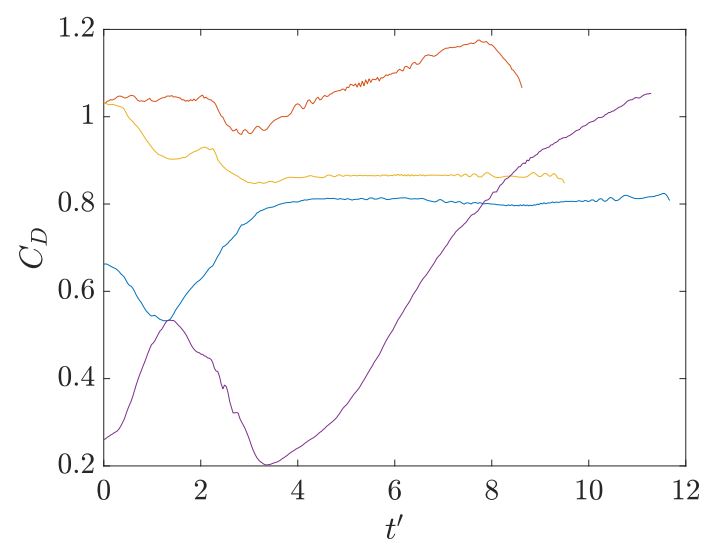

(a)

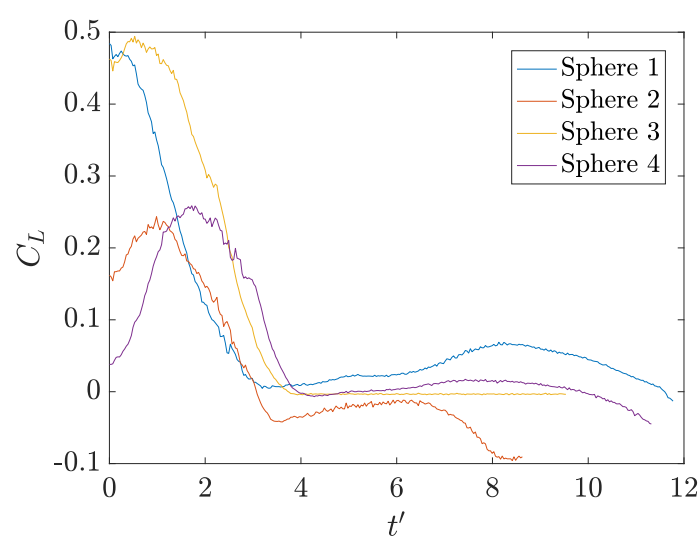

(b)

Fig. 13 (a) Drag coefficient and (b) lateral force coefficient.

teristics of given cluster is the velocity of the system's center of mass. All calculations of individual lateral separation velocity and lateral force coefficient are thus referenced to the kinematics of the nonstationary center of mass, whereas the ensemble motion of the separated cluster is computed by averaging the contributions of all bodies. In the remainder of this work, we simply treat $C$ as a scaling parameter for the collective lateral velocity related by:

$$
\overline{V_{T}}=\sqrt{C \frac{\rho_{\infty}}{\rho_{\mathrm{sph}}}} U_{\infty},
$$

where the dependence on radius ratio has been dropped with the understanding that the results obtained are unique to the selected arrangement of bodies. For the sake of completeness, however, we note that the ratio of sphere radius to the radius of a virtual sphere circumscribing the cluster is 0.45, a factor which can be thought of as being implicitly absorbed into $C$. Alternatively, the ratio compared with a virtual sphere of the same mass as the cluster is 0.63.

In the case of two equal-sized spheres separating, the parameter governing the resultant dynamics is the initial polar alignment angle, and we might likewise expect the behavior of the individual spheres in a tetrahedron cluster to be tightly correlated to their initial positioning. Previously, we considered $\theta$ to be the alignment angle between primary sphere and secondary sphere; in the expanded system, the more logical reference point is the center of mass, so $\theta$ now denotes the polar angle between a sphere's center and the cluster center of mass. Here, we investigate the dependence of ensemble separation behaviors on $\theta_{0}$ by conducting the following procedure. First, considering all spheres in the survey independent of the other bodies present in their constituent simulations, we bin the spheres into groups by initial polar angle, with a bin width of $15^{\circ}$ deemed adequate. Next, we compute the lateral velocity of all bodies relative to their cluster's center of mass before nondimensionalizing. Note that the number density distribution vanishes towards $\theta_{0}$ values of $0^{\circ}$ and $180^{\circ}$, so bins towards the extremes of the range are less populated than those nearer the center. In Figure 14, the stacked timeseries of grouped lateral velocities shows a clear dependence of trajectory on initial polar angle. Spheres positioned towards the front of the formation $\left(0^{\circ}-30^{\circ}\right)$ tend to experience mild separation velocities, likely because the downstream spheres are largely incapable of influencing their dynamics, while those located slightly upstream of the flank $\left(60^{\circ}\right.$ $-90^{\circ}$ ) are subject to immediate expulsion or shock surfing, reaching nondimensional velocities of 0.68. At intermediate forward positions $\left(30^{\circ}-60^{\circ}\right)$, the separation characteristics seem dominated by collisions with other spheres, which are manifested as discontinuous jumps in $V_{T}^{\prime}$ and seem to occur after only a small degree of repulsion. This category is mainly occupied by the leader of a sphere pair repulsed in tandem (as in spheres 1 and 4 in Figure 12), and the enhancement in $V_{T}^{\prime}$ through the two-sphere subcluster interaction can reach values of $\sim 0.2$, which is consistent which the results of Section IV B. Polar angles above $90^{\circ}$ mark a transition from expulsion to entainment trajectories, although the notable variation in separation velocities at $\theta_{0}=90^{\circ}-120^{\circ}$ indicates some level of dependence on geometry-specific properties. Note that, while some bodies seem not to 


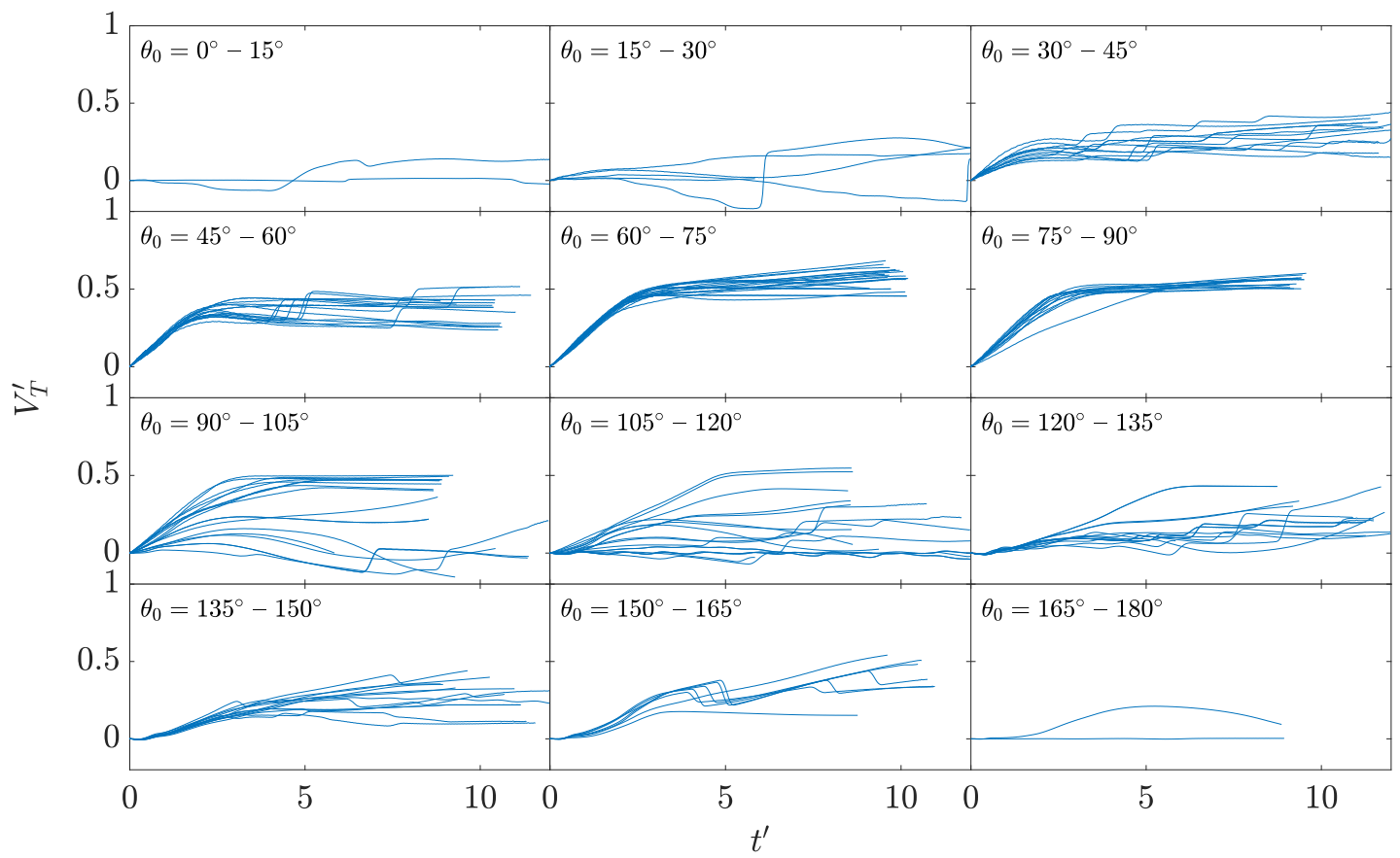

Fig. 14 Timeseries of nondimensional lateral velocity grouped by initial polar angle.

have leveled off to a final lateral velocity, the distribution of kinematics at simulation termination is likely a fairly accurate representation of the true range of values. Just as bins $30^{\circ}-60^{\circ}$ constitute the upstream (primary) bodies in a sphere pair, the spheres in bins $120^{\circ}-165^{\circ}$ represent their downstream (secondary) counterparts in the same manner as described in Section IV B; these, too, are marked by collisions and a delayed increase in lateral velocity typical of the 'rolling' trajectories. Finally, in the rear of the formation, rotational symmetry keeps the lateral separation velocities small.

Using the final lateral velocities of each sphere, we can create a basis for estimating separation velocities based on initial polar angle alone. Defining the final velocity as that attained when a sphere reaches the limits of the computational domain or when the simulation ends, we present the mean lateral velocity of each binned group, along with error bars denoting one standard deviation, in Figure 15a. The lateral separation velocity increases nearly linearly from initial polar angles of $0^{\circ}$ to $\sim 70^{\circ}$, the latter marking the angle at which extended shock surfing is probable. At the lower end of this linear separation regime, the lateral forces on the sphere of interest are minimal, but the dynamical influence of other bodies grows towards higher polar angles where they will be more closely aligned in streamwise positioning and more inclined to generate a common bow shock. The brief plateau between $\sim 70^{\circ}$ and $\sim 90^{\circ}$ is consistent with the high sensitivity of shock surfing to initial positioning and represents the highest degree of expulsion observed in the survey. The nondimensional lateral velocities of 0.6 achieved here are roughly consistent with the measurements of Laurence et al. [9] of the shock surfing behavior of secondary spheres with radius ratio 0.625 from approximately the same range of initial alignment angles. A substantial reduction in separation velocity occurs thereafter as a result of the increasing number of entrainment events, although a wide spread in the data is apparent. Above $\theta_{0}=120^{\circ}$, we again observe a roughly linear rise in the separation velocity; these bins represent spheres that are initially shielded from the freestream flow by an upstream body and remain in the wake of that body until a nominal two-sphere interaction can commence, so it is not entirely surprising the trends here seem to mirror those at lower $\theta_{0}$ values. Based on the geometric properties of a regular tetrahedron, secondary spheres at larger polar angles are shielded by spheres on the higher angle end of the primary linear regime, resulting in positive linear slopes in both instances. In general, the response of secondary spheres to repulsion is delayed (see Figure 14), which causes the 'rolling' action to occur towards the cluster's center of mass and consequently accounts for a reduced separation velocity relative to that of the primary 


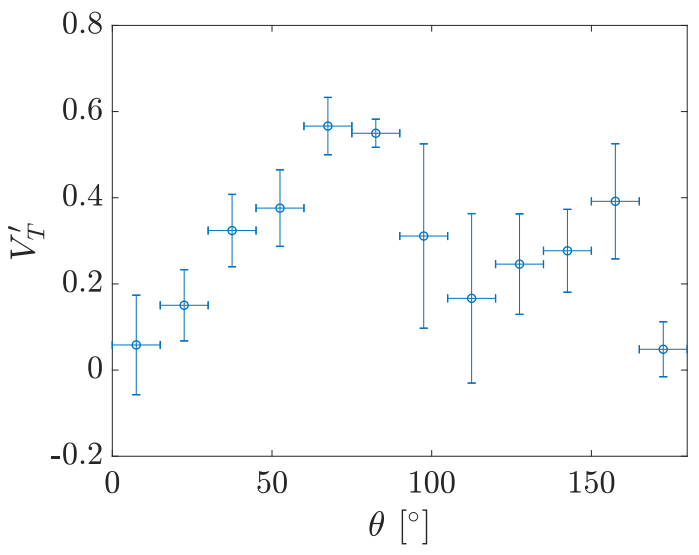

(a)

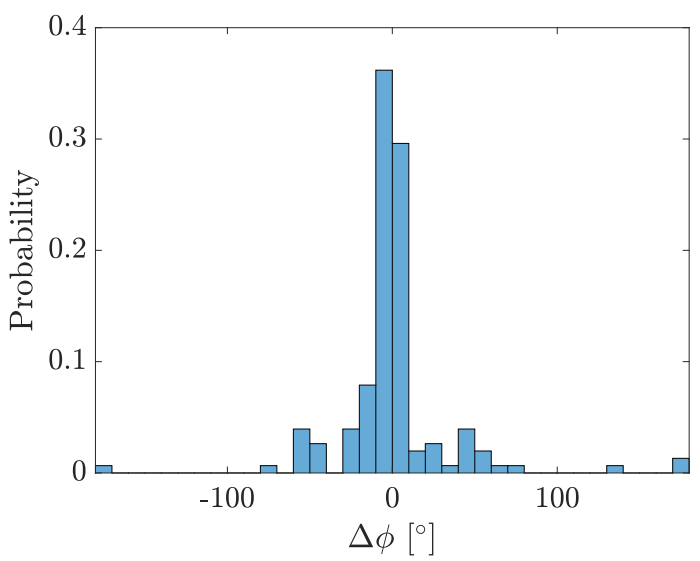

(b)

Fig. 15 Ensemble statistics examining (a) terminal nondimensional lateral velocity binned by initial polar angle and (b) difference between final and initial azimuthal angle.

sphere. Close to $180^{\circ}$, tetrahedron symmetry appears to keep the separation velocity low, but the two-sphere sample size is too small to draw any reliable conclusions. Because most of the variation in separation velocity seems to be captured by the polar angle, it is plausible that reduced-order statistical modeling of separation could accurately predict the terminal trajectory of constituent fragments without specifically treating the geometrical intricacies of the initial cluster. Although we have not yet considered clusters with larger numbers of fragments, we expect similar physical effects to be dominant, and thus that the qualitative trends observed here may persist.

Existing models of the aerodynamic separation of fragmenting bodies (the pancake model [7], for example) assume pure radial expansion of the free-flying bodies. Such a simplification has not been tested, however, using computational fluid dynamic tools. As a simple test of this assumption, we investigate the difference in azimuthal angle, $\phi$, over the course of a simulation, where $\phi$ is referenced to the position of the cluster center of mass. Figure 15b shows that the majority of spheres do not experience much change to their azimuthal positions: $75 \%$ remain with $20^{\circ}$ of their initial positions, while a handful of entrained bodies have passed through the wake of a primary sphere to achieve $\Delta \phi$ values of $\pm 180^{\circ}$. A moderate number of bodies have moved further from their initial azimuthal positions, though tetrahedral symmetries are likely a contributing factor, as such cases tend to occur with pairs of trailing spheres near initial yaw angles of $0^{\circ}$. Despite some variation in azimuthal angle as a result of a separation event, we find that the preponderance of bodies do expand nearly laterally outward from their initial positions, justifying to some extent the use of that assumption in simplified aerodynamic modeling of fragmentation events.

Now that we have established some details regarding the separation behavior of spheres based only on initial positioning in a cluster, we can examine the characteristics of the clusters as determined by their orientations. The survey parameters subject to variation were the pitch and yaw of the tetrahedral arrangement; while these parameters do drive the range of behaviors achieved, they do not possess an underlying physical correlation with the separation characteristics of a given cluster. Instead, we derive a set of reduced parameters that are functionally independent of the specific geometry considered and provide some degree of insight into the expected collective trajectory of the cluster. First, we define a bluntness parameter, $B$, which effectively describes whether the primary bow shock is generated by multiple spheres, thus creating a region of high pressure between the bodies and promoting separation. For a given tetrahedron orientation, $B$ is determined by computing the unit normals for all external faces and finding the maximum projection onto the upstream direction:

$$
B=\max \left(\hat{n}_{i} \cdot\langle-1,0,0\rangle\right) .
$$

The geometry of tetrahedron limits the bluntness index to $[1 / 3,1]$, where larger values indicate a higher proclivity for immediate repulsion. The second parameter is the asymmetry index, which represents the lateral mass offset between the aerodynamically relevant bodies and the system's 


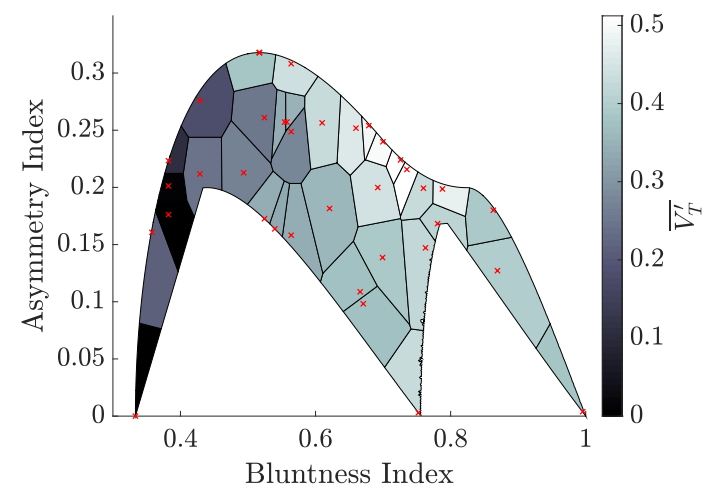

(a)

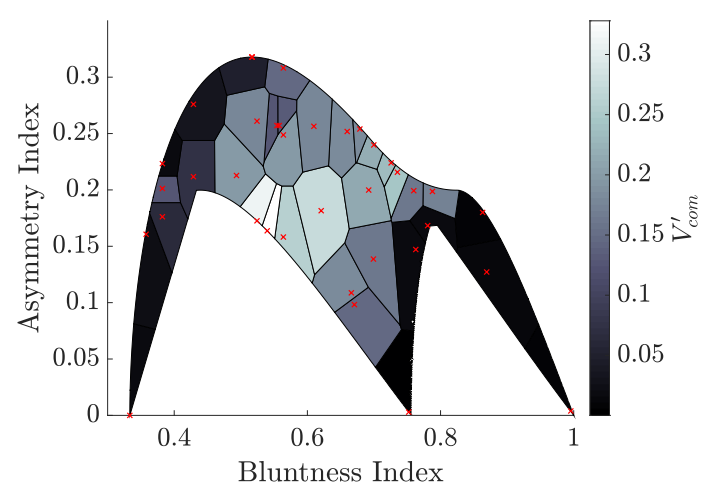

(b)

Fig. 16 Maps of nondimensional (a) collective separation velocity and (b) lateral center of mass velocity under the reduced parameterization.

center of mass. To compute this quantity, we determine the initial lateral distance of each sphere from the center of mass and assign a summation weight based on its streamwise position; a quadratic weighting is utilized upstream of the center of mass, while downstream spheres are assigned a value of zero. The set of weights is normalized to unity, and the 2-norm of the summation is scaled by the radius of the virtual circumscribing sphere:

$$
A=\left\|\Sigma_{i} w_{i}^{\prime}\left(\vec{x}_{i, l}-\vec{x}_{\mathrm{com}, l}\right)\right\| / r_{v}, \quad \text { where } \quad w_{i}=\left\{\begin{array}{ll}
\left(x_{i}-x_{\mathrm{com}}\right)^{2} & , x_{i}<x_{\mathrm{com}} \\
0 & , x_{i} \geq x_{\mathrm{com}}
\end{array} \quad \text { and } \quad w_{i}^{\prime}=\frac{w_{i}}{\Sigma_{j} w_{j}}\right.
$$

Here, the $l$ subscript refers to the lateral component the position vector and $r_{v}$ is the virtual sphere radius. The asymmetry index takes values between 0 and $\sim 0.32$ for a tetrahedron, where the aerodynamic offset from the primary axis is greatest at larger values. The locus of points in the $A-B$ plane is highly dependent on the cluster geometry and appears much more irregular in form than does the rectangular pitch-yaw space explored for survey purposes. Furthermore, we note that this set of parameters maps roll-degenerate states to the same coordinates in $A-B$ space.

In Figure 16, we present maps of the surveyed cluster separation properties under the reduced parameterization. With the boundary derived from a random sampling of cluster orientations and simulation points noted in red, the vertex-centered tessellations give the nondimensional collective separation velocity of the cluster, $\overline{V_{T}^{\prime}}$, and the lateral velocity of its center of mass, $V_{\text {com }}^{\prime}$ In the current context, Figure $16 \mathrm{a}$ can also be considered a representation of $\sqrt{C}$. The map of $\overline{V_{T}^{\prime}}$ demonstrates a clear correlation of collective separation velocity with the bluntness index. Near $B$ values of $1 / 3$ (i.e., more streamlined cases), the cluster approaches separation velocities of zero; by extrapolation of the results of Section IV B, one might expect a lower limit of $\sim 0.2$, but certain arrangements possess symmetries that render them somewhat resistant to separation. In Figure 17a, for example, increased outboard pressures on the rear spheres due to shock impingement are balanced by contact forces, allowing the cluster to translate downstream without any perceptible disruption. Such behavior may not be characteristic of actual entry events, however. Towards higher values of $B, \overline{V_{T}^{\prime}}$ increases to $\sim 0.5$, which tends to occur when all spheres are immediately repulsed. While one might expect little dependence of the collective separation velocity on the asymmetry index, we find that a larger aerodynamic offset can enhance separation compared to initially blunter clusters with high symmetry. In Figure $17 \mathrm{~b}$, the presence of one trailing sphere along the primary axis reduces the overall lateral velocity of the cluster, while all spheres (including a primary/secondary pair) are repulsed from the center of mass in Figure 17c. There is clearly a high degree of variation in the collective separation velocities experienced by a tetrahedral cluster of spheres, but we attempt to estimate a characteristic $\overline{V_{T}^{\prime}}$ for comparative purposes. Weighting each simulation by its normalized cell area in Figure 16a, we find a value of $\sim 0.34$, which corresponds to a $C$ of $\sim 0.12$. Although this is somewhat lower than other estimates of this quantity, it does compare favorably with the $C$ of 0.2 in Artem'eva and Shuvalov [3] when scaled by the radius ratio of a virtual circumscribing sphere.

From Figure 16b, the cluster center of mass can deviate significantly from the initial primary 


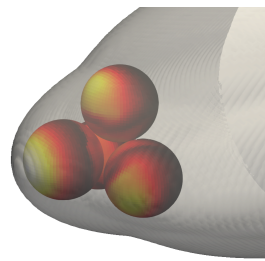

(a)

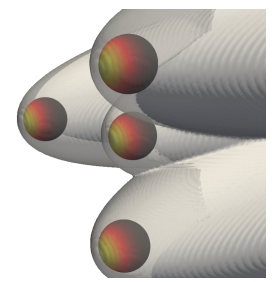

(b)

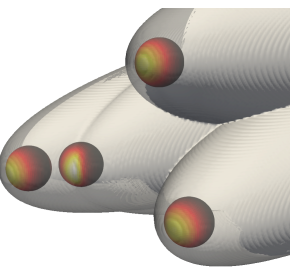

(c)

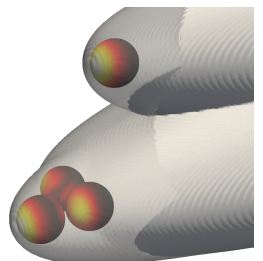

(d)

Fig. 17 Simulation snapshots of cases with asymmetry-bluntness indices of (a) 0-0.33, (b) $0-1$, (c) $0.73-0.22$, and (d) 0.52-0.17.

axis; the area-weighted mean of the survey comes to a value of $\sim 0.13$, large enough to meaningfully alter the trajectory and location of energy deposition due to a body entering the atmosphere. The correlation with the asymmetry index is not so clear here, however. Highly symmetrical cases (see Figures 17a and 17b) do tend towards a laterally stationary center of mass, but the most severe instances of center of mass repulsion occur for only moderately asymmetric agglomerations. For instance, Figure 17d shows a quasi-stable subcluster of three spheres that skews the center of mass sharply away from its initial position. At higher $A$ values (Figure $17 \mathrm{c}$ ), primary/secondary sphere pairs can generate transient lifting configurations before eventually separating, although, at $A=0.31$, the lateral forces induced by a streamwise-aligned sphere pair are small enough that their lateral momentum is almost completely offset by two bodies initially expelled from the cluster. The acute shift of mass away from the nominal trajectory axis may be a peculiarity of the current geometry. Indeed, as the number of spheres is increased, one might expect more uniform spreading of the constituent bodies in a cluster with little offset to the center of mass location. In the current framework, such a situation would equate with reducing the extent of the explorable asymmetrybluntness space.

\section{Conclusions}

In this study, we conducted numerical simulations investigating the separation of clustered spheres in inviscid Mach 20 flow. AMROC/DYNA3D, the coupled CFD/FEA solver capable of handling collisions between bodies, was compared against a validating free-flight experiment from the University of Maryland's Mach 6 HyperTERP facility. Lack of viscosity modeling was deemed to have considerably altered the trajectory of one sphere in the cluster, but agreement in lateral separation velocities was close enough to consider the results of AMROC/DYNA3D characteristic of sphere separation behaviors in general. A survey of two initially touching spheres of equal sizes examined separation behavior over a range of polar alignment angles. Based on rigid-body modeling of the pair, a stable region centered about $140^{\circ}$ in which the spheres resist aerodynamic separation was discerned. Furthermore, at higher alignment angles, the downstream sphere tended to 'roll' towards the side of the upstream sphere, its momentum carrying it through the stable regime, and eventually separated with a nondimensional lateral velocity of 0.2 , indicating a bimodal distribution of separation behaviors. A survey of four equal spheres placed at the vertices of a regular tetrahedron demonstrated a well-defined set of trajectories strongly correlated to the initial polar angle of a given body. A set of reduced parameters evaluating the bluntness and asymmetry of a sphere arrangement revealed a range of collective lateral velocities and potentially significant shifts of the cluster center of mass away from its initial primary axis. This arrangement of spheres exhibited an aerodynamic separation coefficient, $C$, of $\sim 0.12$, which is lower than other estimates of this parameter but does not include a radius ratio scaling.

\section{Acknowledgments}

T. J. Whalen was supported by a NASA Space Technology Research Fellowship. 


\section{References}

[1] Mathias, D. L., Wheeler, L. F., and Dotson, J. L., "A probabilistic asteroid impact risk model: assessment of sub-300 m impacts," Icarus, Vol. 337, 2017, pp. 106-119. doi: https://doi.org/10.1016/j.icarus.2017.02.009.

[2] Passey, Q. and Melosh, H., "Effects of Atmospheric Breakup on Crater Field Formation," Icarus, Vol. 42, 1980, pp. 211-233. doi:https://doi.org/10.1016/0019-1035(80)90072-X.

[3] Artemieva, N. and Shuvalov, V., "Interaction of Shock Waves during the Passage of a Disrupted Meteoroid through the Atmosphere," Shock Waves, Vol. 5, 1996, pp. 359-367. doi: https://doi.org/10.1007/BF02434011.

[4] Artemieva, N. and Shuvalov, V., "Motion of a Fragmented Meteoroid through the Planetary Atmosphere," Journal of Geophysical Research, Vol. 106, No. E2, 2001. doi: https://doi.org/10.1029/2000JE001264.

[5] Laurence, S. and Deiterding, R., "Shock-Wave Surfing," Journal of Fluid Mechanics, Vol. 676, 2011, pp. 396-431. doi:https://doi.org/10.1017/jfm.2011.57.

[6] Wheeler, L., Register, P., and Mathias, D., "A Fragment-Cloud Model for Asteroid Breakup and Atmospheric Energy Deposition," Icarus, Vol. 295, 2017, pp. 149-169. doi: https://doi.org/10.1016/j.icarus.2017.02.011.

[7] Hills, J. G. and Goda, M. P., "The Fragmentation of Small Asteroids in the Atmosphere," The Astronomical Journal, Vol. 105, No. 3, 1993, pp. 1114-1144. doi:https://doi.org/10.1086/116499.

[8] Register, P. J., Aftosmis, M. J., Stern, E. C., M., B. J., Seltner, P. M., Willems, S., Guelhan, A., and Mathias, D. L., "Interactions between asteroid fragments during atmospheric entry," Icarus, Vol. 337, No. 113468, 2020. doi:https://doi.org/10.1016/j.icarus.2019.113468.

[9] Laurence, S., Parziale, N., and Deiterding, R., "Dynamical Separation of Spherical Bodies in Supersonic Flow," Journal of Fluid Mechanics, Vol. 713, 2012, pp. 159-182. doi: https://doi.org/10.1017/jfm.2012.453.

[10] Deiterding, R., "A Parallel Adaptive Method for Simulating Shock-Induced Combustion with Detailed Chemical Kinetics in Complex Domains," Computers and Structures, Vol. 87, 2009, pp. 769-783. doi: https://doi.org/10.1016/j.compstruc.2008.11.007.

[11] Deiterding, R. and Wood, S., "Parallel Adaptive Fluid-Structure Interaction Simulation of Explosions Impacting on Building Structures," Computers and Structures, Vol. 88, 2013, pp. 719-729. doi: https://doi.org/10.1016/j.compfluid.2013.05.009.

[12] Whirley, R. G. and Engelmann, B. E., "A nonlinear explicit three-dimensional finite element code for solid and structural mechanics," Tech. Rep. UCRL-MA-107254-Rev.1, Lawrence Livermore National Laboratory, 1993. doi:https://doi.org/10.2172/10139227.

[13] Deiterding, R., Radovitzky, R., Mauch, S. P., Noels, L., Cummings, J. C., and Meiron, D. I., "A virtual test facility for the efficient simulation of solid material response under strong shock and detonation wave loading," Engineering with Computers, Vol. 22, 2006, pp. 325-347. doi:https://doi.org/10.1007/s00366006-0043-9.

[14] Mauch, S. P., Efficient algorithms for solving static Hamilton-Jacobi equations, Ph.D. thesis, California Institute of Technology, 2003.

[15] Butler, C. S. and Laurence, S. J., "HyperTERP: A newly commissioned hypersonic shock tunnel at the University of Maryland," AIAA Aviation Forum, June 2019. doi:https://doi.org/10.2514/6.2019-2860, AIAA Paper 2019-2860.

[16] Laurence, S. and Karl, S., "An Improved Visualization-Based Force-Measurement Technique for Short-Duration Hypersonic Facilities," Experiments in Fluids, Vol. 48, 2010, pp. 949:965. doi: https://doi.org/10.1007/s00348-009-0780-9. 